\title{
HEAT FLUX JUMP METHOD AS A POSSIBILITY OF CONTACTLESS MEASUREMENT OF LOCAL HEAT TRANSFER COEFFICIENTS
}

\author{
Stanislav Solnař*, Martin Dostál \\ Czech Technical University in Prague, Faculty of Mechanical Engineering, Department of Process Engineering, \\ Technická 4, 16607 Prague, Czech Republic \\ * corresponding author: stanislav.solnar@fs.cvut.cz
}

\begin{abstract}
This article deals with the derivation of the temperature response of the finite thickness wall, which is heated by heat flow, as a possibility to measure the local values of the heat transfer coefficient with a very high spatial resolution. In addition to its own experimental measurement technique, it is also possible to use this derivation to describe the gradual heating of the wall while using the temperature oscillation (TOIRT) method for the heat transfer measurement. The system of the partial differential equations and the boundary and initial conditions is solved by a transformation into Laplace's space, where the task is easier to solve. At the end of the article, the results of the analytical solution are compared with a numerical solution of this problem.
\end{abstract}

KEYworDs: Heaviside function, heat transfer coefficient, experimental method, heat jump method.

\section{INTRODUCTION}

When designing heat transfer devices, the heat transfer surface is a critical parameter. The size of the heat transfer surface directly affects the amount of the heat flow passing through these devices. The relationship between these variables is described by Newton's law of cooling

$$
\dot{Q}=\alpha S \Delta T \text {. }
$$

The temperature difference $\Delta T$ is mostly given by the requirements (depending on the avaiable steam or water temperature, the maximum overheating in the food industry ...) and thus the only variable that can affect the size of the heat transfer surface $S$ for the desired heat flux $\dot{Q}$ is the heat transfer coefficient $\alpha$. The heat transfer coefficient represents the intensity of the transferred heat and is dependent on the properties of the liquid and, above all, the relative speeds of the solid wall and the fluid. The heat transfer coefficient is most often measured by experiments, or in the present time computer-aided by numerical simulations, which, however, need an experimental (or theoretical, if possible) validation of the calculated data.

The most commonly used method for measuring the heat transfer coefficient is the static method of measuring the wall surface temperature. A thin plate or foil is fixed in place and most often heated by electric current and is affected by the convective heat transfer we deliberately evoke. Heat transfer coefficients cause a temperature field to form on the thin plate, which can then be used to calculate local heat transfer coefficient values. This method was used, for example, by Katti and Prabhu [1], which measured the heat transfer between the smooth plate and the air flow from the nozzle, and used the infra-red camera to measure the local surface temperatures. Kenneth et al. 22 used an adiabatic wall to measure the heat transfer between smooth wall and a matrix of impinging jets. The smooth wall was coated with a thin layer of thermochromic liquid crystals (TLC, a thermally active colour that changes its colour when changing the temperature). The wall with TLC layer was scanned by a RGB camera and the recorded colour at each point was recalculated to a temperature field. Quite the same method, but another way of measuring the surface temperature field. Beniaiche et al. 3 measured with a help of the stationary method, the heat transfer in the trailing edge of the modern gas turbines. The 30:1 replacement model was coated with a thin calibrated TLC layer and RGB camera scanned the temperature field while the cooling air was flowing. The pressure field was also studied. The resulting heat transfer coefficient maps are used to optimize the trailing edges and prevent overheating of the modern turbine blades.

Frequently used heat transfer measurement methods are the transient methods of a surface temperature measurement. Hippensteele and Poinsatte [4] measured the heat transfer coefficient maps in a tapered channel for high Reynolds numbers $\left(2 \times 10^{5}\right.$ to $\left.1.2 \times 10^{6}\right)$. Authors pre-warmed the measured channel, and after reaching the initial temperature throughout the model volume, they began to cool the channel through the internal air stream and recorded the surface of the channel with a TLC calibrated layer by RGB camera.

Another, very often used method, is the electrodiffusion method (EDM), which measures the current between two electrodes. When measuring with this method, it is necessary to have two electrodes and an electrolytic 
liquid. If we bring the voltage to electrodes, a heterogeneous reaction results in a change of equalization between anode and cathode, which induces a measureable current. The stronger the mass transfer of the ions, the higher are the measured values of the current [5]. This method for measuring local values of the heat transfer coefficient was used, for example, by Petera et al. [6] in the measurement of the local heat transfer coefficients at the bottom of the agitated vessel on which the jet of water was falling. The impinging jet was formed by an agitator that was placed in the draft tube. Another application can be, for example, Cudak and Karcz [7], where the heat transfer coefficients were measured on the wall of the agitated vessel without baffles and the agitator had been placed in the axis or outside the axis of the agitated vessel. Karcz a Strek [8] also measured the influence of the baffles, their location and the length of the heat transfer on the wall of an agitated vessel with this method.

A relatively new and modern method for measuring the local heat transfer coefficient values is the temperature oscillation infra-red thermography method (TOIRT). This method is based on measuring the surface temperature of the wall with infra-red camera, which is influenced by the oscillating heat flow from one side and the convective heat transfer from both sides of the wall. The key information of the method is the phase delay of the temperature signal behind the oscillating heat flux, this delay is in a direct relationship with the heat transfer coefficient. Authors Wandelt and Roetzel [9] derived this relation and tried to measure the heat transfer on a wall that had an air flow from both sides as well. By comparing the experimental results with the assumed values at the individual measurement points, the functionality of the method was confirmed.

Freund [10] subjected this method to a very thorough theoretical analysis and set certain practical limits (maximum and recommended wall thickness, optimal oscillating heat signal frequency, etc.) for the method to function. The thesis also presents a sensitivity analysis of the method to input variables, the theoretical and experimental validation and the problems associated with the method. Besides the experiments themselves, he also dealt with the problem of lateral conduction in the wall that occurs during certain measurements, but the method does not take this into account. The author tries to solve this problem by means of a back-up task and a numerical finite-element method modelling. Freund also encountered the problem of the gradual heating of the entire system (he called that drift), which arises at the beginning of the measurement as a temperature transient response. This gradual warming of the measured wall must be removed from the data if we want to use all measured data. In a theoretical level, we should wait an infinitely long time to guarantee the thermal equilibrium of the system, which is not entirely enjoyable from a practical point of view. By removing the drift from the data, it is possible to use the data that do not meet the thermal equilibrium condition. This temperature tendency caused a relatively large error in the values of the temperature phase delay (which are in relation to the resulting heat transfer coefficient), and Freund removed it by interleaving the various functions and subtracting them and claimed that this procedure did not have a significant effect on the method error.

Freund published several papers on the application of the TOIRT method eg. [1] when measuring the local values of the heat transfer coefficient in the plate heat exchanger plates for which he prepared the numerical model in ANSYS Fluent or [12] when measuring heat transfer in water cooling spray systems. In Freund [10, there are also experiments, such as heat transfer in the flow of fluid in the tube, heat transfer behind the vortex generators in the air tunnel, or heat transfer between the plate and the impinging jet experiments.

We will solve this problem using an integral transformation that simplifies the solution of similar problems. The Laplace transformation is an integral transformation named after its discoverer Pierre-Simon Laplace [13. The real variable $t$ function is transformed into the $s$ complex variable function, which can be transformed back.

Laplace transformation $\mathcal{L}$ transforms the functions of the real variable $f(t)$ defined for all real numbers $t \geq 0$ to the images of the functions $F(s)$ defined as

$$
\mathcal{L}(f(t))=\int_{0}^{\infty} f(t) e^{-s t}=\bar{f}(s)
$$

We will use $\bar{f}$ for function images in Laplace's space. The $f(t)$ function must be locally integrable on the $[0, \infty)$ interval. In the literature, it is then possible to find images (Laplace transformations) of many different functions.

Laplace transform is also very often used to solve differential equations (both ordinary differential equations and partial differential equations). If we transform an ordinary derivative of the function $f^{\prime}(t)$

$$
\mathcal{L}\left(\frac{\mathrm{d} f(t)}{\mathrm{d} t}\right)=\mathcal{L}\left(f^{\prime}(t)\right)=s \bar{f}(s)-f(0)
$$

we get an image of a function that is in the form of an algebraic expression. The system of such equations can then be solved very easily and the solution of the equation is obtained by the inverse transformation. If we transform the partial derivation of function $f(x, t)$ by time, we get a function image

$$
\mathcal{L}\left(\frac{\partial f(x, t)}{\partial t}\right)=s \bar{f}(x, s)-f(x, 0),
$$




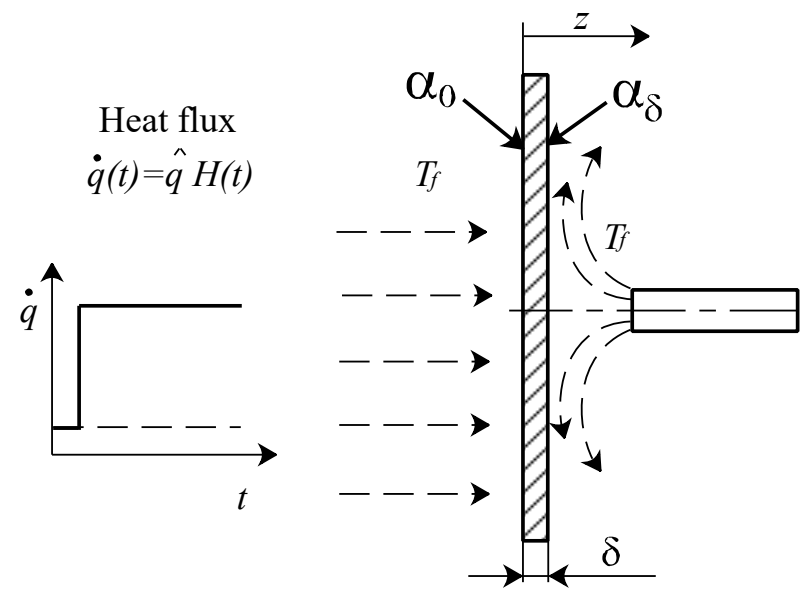

FiguRE 1. A schematic drawing of the new method applied to a wall with a finite thickness $\delta$ and further influenced by the heat transfer coefficiens $\alpha_{0}$ and $\alpha_{\delta}$.

which is again in the form of an algebraic expression. For example, if we transform the second partial derivative of $f(x, t)$ by the coordinate of $x$ we get

$$
\mathcal{L}\left(\frac{\partial^{2} f(x, t)}{\partial x^{2}}\right)=\frac{\mathrm{d}^{2} \bar{f}(x, s)}{\mathrm{d} x^{2}}
$$

which is an ordinary differential and so the solution is simplified to an ordinary differential equation. Partial differential tasks are simplified in Laplace's space to ordinary differential tasks, which are much easier to solve, and we can get their solutions by the inverse transformation.

Numerical solvers of partially differential equations are nowadays included in commercial software, which allow a relatively simple solving of these tasks. For 1D tasks, MATLAB offers the pdepe function that solves the initial tasks of parabolic and elliptical equations in a one selected direction. After defining the task, boundary and initial conditions, and the number of time and dimension points, we get the result within a few seconds. A detailed description of the use of this function can be found at https://www.mathworks.com/help/matlab/ ref/pdepe.html.

Probably the most commonly used tool for solving differential problems is ANSYS Fluent. It seems unnecessary to use such a powerfull tool to solve the banal problem of heat conduction in a homogeneous wall.

\section{DeFinition OF THE PROBLEM}

The idea of the new heat flux jump method is depicted on the Fig. 1

It is a wall that has infinite dimensions in the direction of $x$ and $y$, and in the direction $z$ has a finite thickness of $\delta$, which is assumed to be very small (in milimeters). This wall with known thermophysical properties (density $\rho$, specific heat capacity $c_{p}$ and thermal diffusivity $\lambda$ ) is heated from the left side by a uniform heat flux $\dot{q}$. The heated wall also causes a flow in the region near to the wall, causing a natural convection to the left of the task expressed as the convective heat transfer coefficient $\alpha_{0}$. The temperature field in the wall is also affected by the convective heat transfer coefficient $\alpha_{\delta}$ on the right hand side that we are trying to determine. Heat losses to the enviroment are not considered in this task. The main temperature change is caused by the heat flow, which is applied to the left side of the wall. This heat flow will have a jumping character and can be expressed using Heaviside function $H(t)$.

$$
\dot{q}(t)=\hat{q} H(t),
$$

where $\hat{q}$ represents the amplitude of the heat flux.

Heat conduction in a homogeneous wall is descibed by the Fourier equation.

$$
\frac{\partial T}{\partial t}=a\left(\frac{\partial^{2} T}{\partial x^{2}}+\frac{\partial^{2} T}{\partial y^{2}}+\frac{\partial^{2} T}{\partial z^{2}}\right)
$$

where $a$ represents a temperature diffusivity. Assuming that the incident heat flow to the wall is uniform and that the heat transfer coefficient on the right hand side $\alpha_{\delta}$ does not change on the wall, it can be assumed that the task becomes a 1D problem (there will be no heat conduction in the direction of $x$ and $y$ ), and therefore, 
the first two partial derivatives on the right hand side can be ommited. If we do not calculate the absolute temperature values, but only the change from the initial value $T_{0}$, we can formulate this task as follows:

$$
\frac{\partial\left(T-T_{0}\right)}{\partial t}=a \frac{\partial^{2}\left(T-T_{0}\right)}{\partial z^{2}} .
$$

The boundary condition for solving this task from the right hand side (at the point $z=\delta$ ) is given only by the investigated heat transfer coefficient $\alpha_{\delta}$ and can be formulated as a condition of the third kind:

$$
-\left.\lambda \frac{\partial\left(T-T_{0}\right)}{\partial z}\right|_{z=\delta}=\left.\alpha_{\delta}\left(T-T_{f}\right)\right|_{z=\delta},
$$

where the temperature $T_{f}$ is the ambient temperature. The boundary condition from the left hand side of the wall (at the point $z=0$ ) is given by the heat transfer coefficient $\alpha_{0}$ and by the heat flux that is sent to the measured wall, it, again, is a condition of the third kind and is defined as:

$$
-\left.\lambda \frac{\partial\left(T-T_{0}\right)}{\partial z}\right|_{z=0}=\hat{q} H(t)-\left.\alpha_{0}\left(T-T_{f}\right)\right|_{z=0} .
$$

Since this is a non-stationary task, it is necessary to add the initial condition to these equations. We have now assumed that the initial temperature $T_{0}$ is identical to the ambient temperature $T_{f}$, i.e. the system was originally in the state of a thermodynamic equilibrium, we see from equations (8-11) that $T_{0}$ (or $T_{f}$ ) is only the additive constant and equations $(8-11$ ) we can formulate for

$$
T_{0}=T_{f}=0
$$

regardless of the generatily of the problem. This initial condition will simplify the equations as well as the whole solution.

\section{PROBLEM SOLVING}

We solved this problem in the subsections: General solution, Application of boundary conditions and the Particular solution.

\subsection{General SOlution}

To solve this system of a partial differential equation (8), two boundary conditions (9), 100 and initial condition (11), we first transformed the system into a partial dimensionlessness for a simpler solution. As the first transformation, we converted the $z$ coordinate and defined the dimensionless coordinate $z^{\prime}$ as

$$
z^{\prime}=\frac{z}{\delta},
$$

which is very often seen in the literature. Another variable is the time $t$, which we transformed into a dimensionless time $t^{\prime}$ as follows

$$
t^{\prime}=\frac{a t}{\delta^{2}} .
$$

By introducing dimensionless variables into the Fourier heat conduction equation (8), along with zero initial condition, we obtain an expression

$$
\frac{\partial T}{\partial t^{\prime}}=\frac{\partial^{2} T}{\partial z^{\prime 2}} .
$$

By the Laplace's transformation we can convert this equation into the ordinary differential equation in the Laplace's space.

$$
\mathcal{L}\left(\frac{\partial T}{\partial t^{\prime}}\right)=\mathcal{L}\left(\frac{\partial^{2} T}{\partial z^{\prime 2}}\right) .
$$

We find suitable transformations of the expressions in the "dictionary" to create an image $\bar{T}$ of this equation

$$
s \bar{T}\left(z^{\prime}, s\right)-T\left(z^{\prime}, 0\right)=\frac{d^{2} \bar{T}}{d z^{\prime 2}} .
$$

The second expression on the left hand side of this equation represents the temperature $T$ at $z^{\prime}$ and at time 0 . This temperature is already defined by the initial condition $T_{0}=0$ and so the equation will simplify to

$$
\frac{d^{2} \bar{T}}{d z^{\prime 2}}-s \bar{T}\left(z^{\prime}, s\right)=0
$$


This equation is a homogeneous second order differential equation with constant coefficients that can be solved using the characteristics. The characteristic equation is in this case

$$
\lambda_{c}^{2}-s=0
$$

and the roots of equation $\lambda_{c}$ are then equal to

$$
\lambda_{c}= \pm \sqrt{s}
$$

and the general solution of this equation can, therefore, be found in the form of a sum of hyperbolic functions

$$
\bar{T}\left(z^{\prime}, s\right)=C_{1} \sinh \left(\sqrt{s} z^{\prime}\right)+C_{2} \cosh \left(\sqrt{s} z^{\prime}\right)
$$

where $C_{1}$ and $C_{2}$ are constants that must be solved by determined conditions.

\subsection{TRANSFORMATION AND APPLICATION OF BOUNDARY CONDITIONS}

Both boundary conditions must be transformed into the same dimensionless variables as the main equation and the initial condition $T_{0}=T_{f}=0$ applied to them as well as to the main equation.

$$
z^{\prime}=1 \rightarrow \quad-\left.\lambda \frac{\partial T}{\partial z^{\prime}}\right|_{z^{\prime}=1}=\left.\alpha_{\delta} \delta T\right|_{z^{\prime}=1}
$$

and for the left hand side boundary condition

$$
z^{\prime}=0 \rightarrow \quad-\left.\lambda \frac{\partial T}{\partial z^{\prime}}\right|_{z^{\prime}=0}=\hat{q} H(t)-\left.\alpha_{0} \delta T\right|_{z^{\prime}=0} .
$$

If we perform the Laplace's forward transformation, we get images of both boundary conditions

$$
z^{\prime}=1 \rightarrow \quad-\frac{d \bar{T}(1, s)}{d z^{\prime}}=\frac{\alpha_{\delta} \delta}{\lambda} \bar{T}(1, s)
$$

and

$$
z^{\prime}=0 \rightarrow \quad-\frac{d \bar{T}(0, s)}{d z^{\prime}}=\frac{1}{s} \hat{q} \frac{\delta}{\lambda}-\frac{\alpha_{0} \delta}{\lambda} \bar{T}(0, s)
$$

Because boundary conditions are expressed by derivatives on both left hand sides, it is necessary to calculate the derivative of the general solution.

$$
\frac{d \bar{T}\left(z^{\prime}, s\right)}{d z^{\prime}}=C_{1} \sqrt{s} \cosh \left(\sqrt{s} z^{\prime}\right)+C_{2} \sqrt{s} \sinh \left(\sqrt{s} z^{\prime}\right) .
$$

Applying the coordinate $z^{\prime}=0$ to the equation of the general solution we obtain

$$
\bar{T}(0, s)=\underbrace{C_{1} \sinh (0)}_{=0}+C_{2} \underbrace{\cosh (0)}_{=1}
$$

where the individual hyperbolic functions are already expressed by their values. By applying this coordinate to the derivative of the general solution we obtain

$$
\frac{d \bar{T}(0, s)}{d z^{\prime}}=C_{1} \sqrt{s} \underbrace{\cosh (0)}_{=1}+\underbrace{C_{2} \sqrt{s} \sinh (0)}_{=0}
$$

where the values of hyperbolic functions are again expressed. By applying the boundary condition we get

$$
-\frac{1}{s} \hat{q} \frac{\delta}{\lambda}+\operatorname{Bi}_{0} \bar{T}(0, s)=C_{1} \sqrt{s}
$$

For expression $\bar{T}(0, s)$ we can add the solution 26 from the general equation. By assingning the $\bar{T}(0, s)$, we obtain the first equation for solving integration constants.

$$
-\frac{1}{s} \frac{\hat{q} \delta}{\lambda}+\mathrm{Bi}_{0} C_{2}=C_{1} \sqrt{s}
$$

By setting the coordinate $z^{\prime}=1$ to the general equation we get

$$
\bar{T}(1, s)=C_{1} \sinh (\sqrt{s})+C_{2} \cosh (\sqrt{s})
$$




\begin{tabular}{lll}
\hline Variable & Value & Unit \\
\hline$\dot{q}$ & 2000 & $\mathrm{~W} \mathrm{~m}^{-2}$ \\
$\alpha_{0}$ & 3 & $\mathrm{~W} \mathrm{~m}^{-2} \mathrm{~K}^{-1}$ \\
$\alpha_{\delta}$ & 500 & $\mathrm{~W} \mathrm{~m}^{-2} \mathrm{~K}^{-1}$ \\
$z^{\prime}$ & 0 & - \\
$\delta$ & 0.001 & $\mathrm{~m}$ \\
$\lambda$ & 15.0 & $\mathrm{~W} \mathrm{~m}^{-1} \mathrm{~K}^{-1}$ \\
$c_{p}$ & 500 & $\mathrm{~J} \mathrm{~kg}^{-1} \mathrm{~K}^{-1}$ \\
$\rho$ & 7900 & $\mathrm{~kg} \mathrm{~m}^{-3}$ \\
\hline
\end{tabular}

TABle 1. Table of input values for the calculation. The heat transfer coefficient $\alpha_{0}$ corresponds to the free convection on the heated side of the wall (estimated value based on correlations), $\alpha_{\delta}$ is the representative value of the heat transfer coefficient we are trying to find out. Thermophysical properties are taken for stainless steel EN 1.4307 (see https://www.notzgroup.com/media/wysiwyg/PDF/JAS/werkstoffe/1.4307_EN.pdf

Applying this coordinate to the derivatiove of the general solution we obtaion

$$
\frac{d \bar{T}(1, s)}{d z^{\prime}}=C_{1} \sqrt{s} \cosh (\sqrt{s})+C_{2} \sqrt{s} \sinh (\sqrt{s})
$$

and applying the boundary condition

$$
-\operatorname{Bi}_{\delta} \bar{T}(1, s)=C_{1} \sqrt{s} \cosh (\sqrt{s})+C_{2} \sqrt{s} \sinh (\sqrt{s})
$$

The expression $\bar{T}(1, s)$ can be again assigned from 30 and we get the second equation

$$
-\operatorname{Bi}_{\delta}\left[C_{1} \sinh (\sqrt{s})+C_{2} \cosh (\sqrt{s})\right]=C_{1} \sqrt{s} \cosh (\sqrt{s})+C_{2} \sqrt{s} \sinh (\sqrt{s})
$$

\subsection{Particular solution}

This creates two equations of two unknowns $C_{1}$ a $C_{2}$. This system of two equations can be written into matrix

$$
A=\left[\begin{array}{ll}
\sqrt{s} & \mathrm{Bi}_{0} \\
\sqrt{s} \cosh (\sqrt{s})+\mathrm{Bi}_{\delta} \sinh (\sqrt{s}) & \sqrt{s} \sinh (\sqrt{s})+\mathrm{Bi}_{\delta} \cosh (\sqrt{s})
\end{array}\right]\left[\begin{array}{l}
C_{1} \\
C_{2}
\end{array}\right]=\left[\begin{array}{l}
-\frac{1}{s} \frac{\hat{q} \delta}{\lambda} \\
0
\end{array}\right]
$$

and solved, for example, with Cramer's rule for solving the system of equations. Determinant of the matrix $A$ is

$$
\operatorname{det} A=\sqrt{s}\left[\sqrt{s} \sinh (\sqrt{s})+\operatorname{Bi}_{\delta} \cosh (\sqrt{s})\right]-\operatorname{Bi}_{0}\left[\sqrt{s} \cosh (\sqrt{s})+\operatorname{Bi}_{\delta} \sinh (\sqrt{s})\right]
$$

and the constants $C_{1}$ and $C_{2}$ can be calculated from the subdeterminants (using the Cramer's rule)

$$
C_{1} \operatorname{det} A=-\frac{1}{s} \frac{\hat{q} \delta}{\lambda}\left[\sqrt{s} \sinh (\sqrt{s})+\operatorname{Bi}_{\delta} \cosh (\sqrt{s})\right]
$$

and

$$
C_{2} \operatorname{det} A=\frac{1}{s} \frac{\hat{q} \delta}{\lambda}\left[\sqrt{s} \cosh (\sqrt{s})+\operatorname{Bi}_{\delta} \sinh (\sqrt{s})\right]
$$

Therefore, finding a non-stationary temperature profile in the wall, as a particular solution to equations (8)11), can be expressed in Laplace's space as

$$
\bar{T}\left(z^{\prime}, s\right)=\frac{C_{1} \operatorname{det} A}{\operatorname{det} A} \sinh \left(\sqrt{s} z^{\prime}\right)+\frac{C_{2} \operatorname{det} A}{\operatorname{det} A} \cosh \left(\sqrt{s} z^{\prime}\right)
$$

\section{INVERSE LAPLACE TRANSFORMATION}

Since the particular solution is not quite simple to be divided into partial fractions (i.e., a direct translation of the function $\bar{T}$ ), we have chosen a numerical method for the inverse Laplace transform. All the results presented here will be generated for the values specified in the Tab. 1 .

Authors Abate and Whitt [14] introduced a numerical solver for an inverse Laplace transformation and its generalized form and also re-explained its functioning. We have used the Talbot algorithm, which is based on the deformation of Bromwich's integral. To understand the functioning of Talbot's algorithm you can read their fantastic paper [14]. The Talbot inversion algorithm is freely downloadable for MATLAB as a function talbot_inversion ( $f \_s, t, M$ ) and for the input function $f \_s$, vector of computation times $t$ (in our case the 


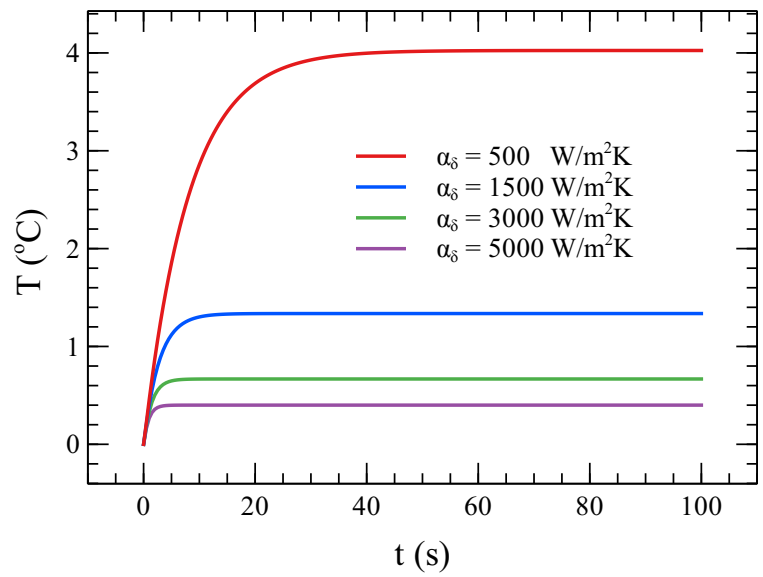

FIgURE 2. The results of the analytical calculation given as a change of temperature $T$ compared to the initial temperature $T_{0}$. The graph is depicted for the values listed in the Tab. 1 and for various values of heat transfer coefficient $\alpha_{\delta}$.

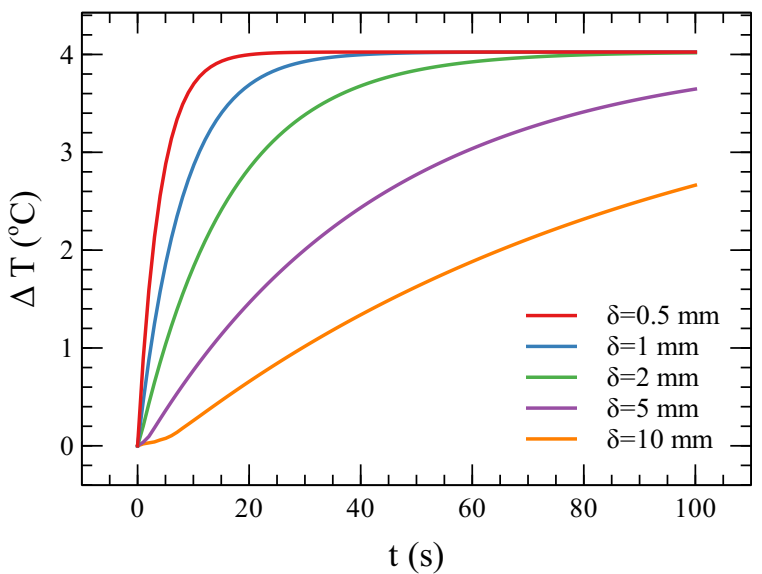

FiguRE 3. Calculated temperature changes for different values of wall thickness $\delta$. The graph is depicted for the values listed in the Tab. 1

dimensionless values of time $t^{\prime}$ ) and the number of iterations of the calculation $M$ returns the real function values in time $f(t)$. The result of this operation applied to our particular solution $\bar{T}\left(z^{\prime}, s\right)$ can be seen in the Fig. 2

The analytically calculated change of temperature $T$ (from the initial temperature $T_{f}=T_{0}=0$, the real temperature of the experiment is only an additive constant) changes in both the time and amplitude domain with the changing value of the heat transfer coefficient $\alpha_{\delta}$. This would mean that, for the evaluation of the heat transfer coefficient, we could theoretically use two pieces of information from the measured data, namely the amplitude of the temperature difference and the time constant of the temperature-time relationship.

The relationship between analytically calculated temperatures and thermophysical properties of the wall or its thickness are depicted in the Fig. 3, 4 and 5 . As we can see, the final temperature, in the long enough time, is not affected by the change of any thermophysical property of the wall or its thickness. The change of these parameters affects the shape of the temperature-time dependence. Thus, a perfect knowledge of the thermophysical properties of the measured wall and its thickness is necessary. The effect of increasing or decreasing heat flux $\dot{q}$ is only in the change of the final temperature. The change of thermal conductivity $\lambda$ makes almost no change to the shape of temperature-time dependence (the maximum error for triple value of thermal conductivity $\lambda$ is $0.019 \mathrm{~K}$ ). The effect of the heat transfer coefficient $\alpha_{0}$ in the range $0-50 \mathrm{~W} / \mathrm{m}^{2} \mathrm{~K}$ is so low that it can almost be neglected.

\section{Numerical SOLUTiON OF THE PROBLEM}

To confirm the correctness of the results, we chose a numerical simulation of this initial problem. For the same conditions $\left(\alpha_{0}, \alpha_{\delta}, \dot{q}, c_{p}, \rho, \lambda, \delta\right.$ a $\left.z^{\prime}\right)$ we have prepared a heat conduction simulation in a homogeneous wall with a finite thickness of $\delta$ and the initial temperature of $T_{0}$ for 20 dimensional points in MATLAB using the 


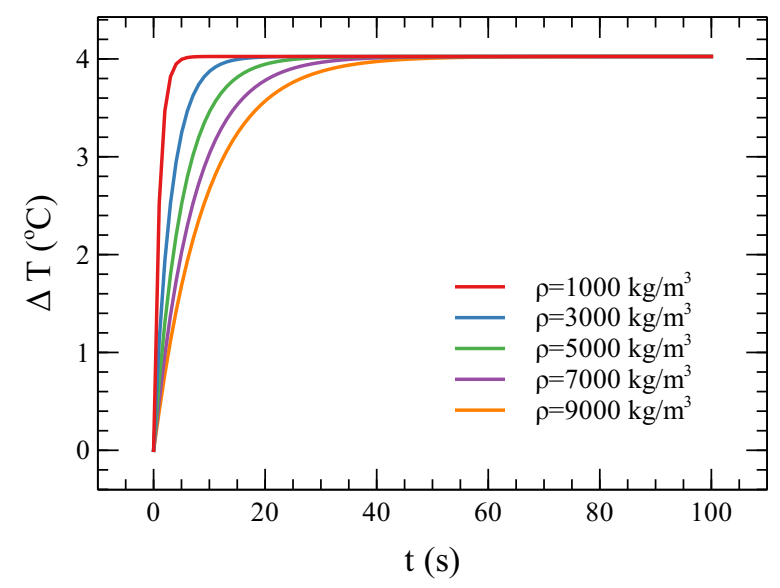

Figure 4. Calculated temperature changes for different values of wall density $\rho$. The graph is depicted for the values listed in the Tab. 1 .

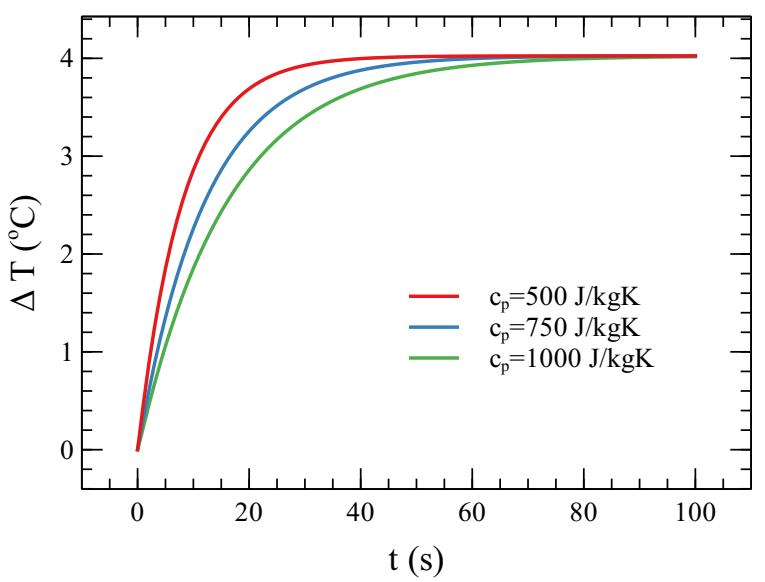

FiguRE 5. Calculated temperature changes for different values of wall heat capacity $c_{p}$. The graph is depicted for the values listed in the Tab. 1

pdepe function. The comparison of calculated changes of the temperature with the analytical method (although with numerical inverse transformation, but analytical) and numerical method can be seen in the Fig. 6 .

\section{Discussion}

We outlined our derivation of the change of temperature in a homogeneous wall with a finite thickness of $\delta$, which is influenced by heat flow rate $\dot{q}$ on one side and convective heat transfer from both sides. Based on the knowledge of this time-temperature curve of the wall and its comparison with the experimentally measured data (i.e. with infra red camera), it is then possible to predict the heat transfer coefficient $\alpha_{\delta}$ on the other side of the wall. A secondary application of this derivation is an analytical description of the gradual heating of the wall by an application of the temperature oscillation method for measuring the heat transfer coefficient.

The analytical calculation shows the possibility of determining the heat transfer coefficient in the temperature domain (each of the heat transfer coefficients belongs to another value of the maximum temperature difference), as well as in the time domain (each of the heat transfer coefficient belongs to another time constant of the temperature progression). The question of experimentally determined values of the heat transfer coefficient will be our future research. This method is independent on the medium in which the heat transfer takes place, as well as the temperature oscillation (TOIRT) method.

Freund [10] described the problems associated with the gradual heating of the wall when applying this method. This exponential part of the temperature dependence made it impossible to correctly determine the phase shift (and hence the heat transfer coefficient) and had to be removed from the data (Freund used a stepwise iterative subtraction of the data from the lines in each period). This calculation could remove this process and describe the exponential part of the temperature dependence better and more accurate. Due to the fact that, at the beginning of the data processing, we will not know the resulting heat transfer coefficient, this process would be probably iterative. 


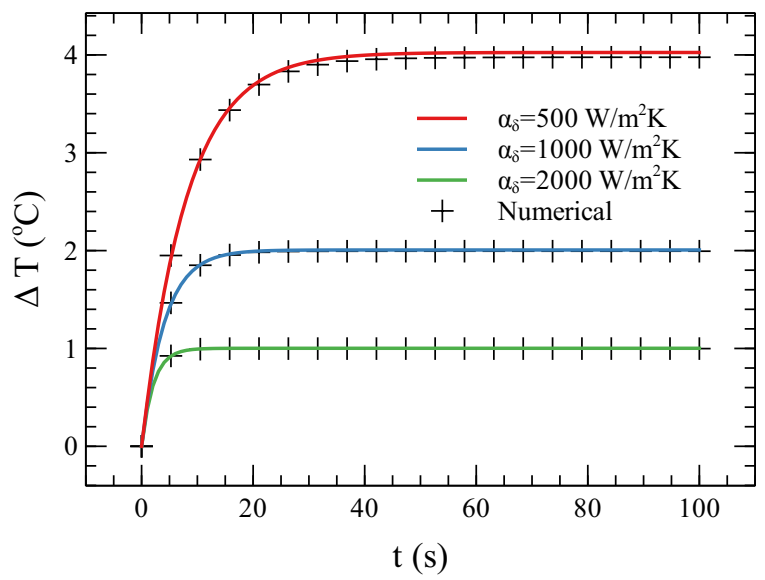

Figure 6. Comparison of analytically and numerically calculated values of temperature changes in the heated wall for various values of heat transfer coefficient $\alpha_{\delta}$.

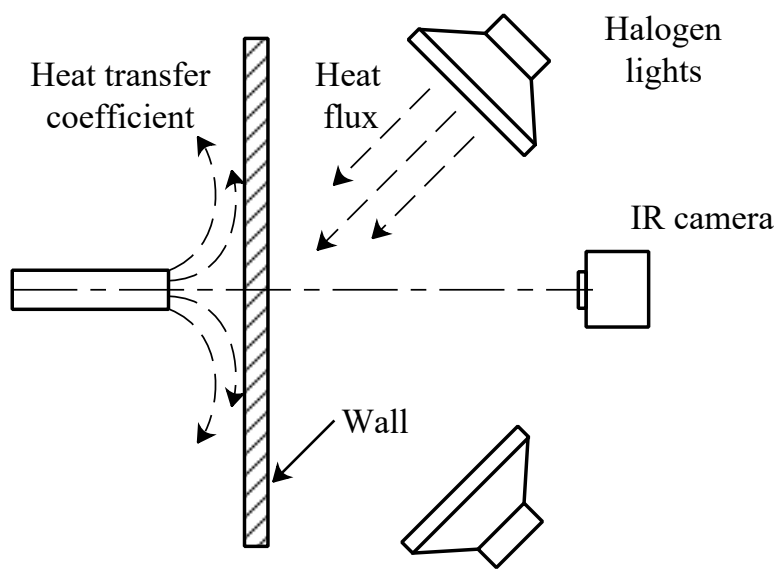

FiguRE 7. Schematic drawing of our idea of experimental measurement.

The inverse transformation of the particle solution using the Talbot algorithm lasts for 200 time steps and number of iterations $\mathrm{M}=64$ (a preset value) about 0.3 seconds.

The results of the analytical calculation were compared to the $1 \mathrm{D}$ heat conduction numerical simulation using the MATLAB software and the pdepe function for 20 dimension points. The results show a very good match of calculated values (the maximum value of the difference is $0.062{ }^{\circ} \mathrm{C}$ ) caused by the numerical implementation of the solved problem.

\section{IDEA OF EXPERIMENTAL MEASUREMENTS}

Our idea of an experimental measurement of local values of the heat transfer coefficient is depicted in Fig. 7 .

A measured wall of metallic material (steel, stainless steel, copper, aluminium ....) is influenced by the heat transfer coefficient we produce (i.e. airflow impinging jet, air flow along the wall, impact fluid flow in the agitated vessel ...). Heat flux is supplied through halogen floodlights, which have a very poor efficiency and much of the power is transformed into heat. These halogen lights will be powered by power source and controlled by a synchronized 2-channel signal. The first signal controls the halogen flood lights and the other triggers the IR camera at specified intervals. Both signals can be generated by a function generator or through a PC card.

The measured data could be then compared with the calculated analytical results (see Fig. 8) and the optimization algorithm will be looking for the best match of analytical and experimental values that will lead to the evaluation of the heat transfer coefficient.

\section{Conclusions}

In this paper, we have presented the derivation of the temperature response of the finite thickness wall, which is affected by the heat flow and also by the convective heat transfer coefficient. After defining the problem, we introduced the equation, boundary and initial conditions that describe the problem. To facilitate the solution 


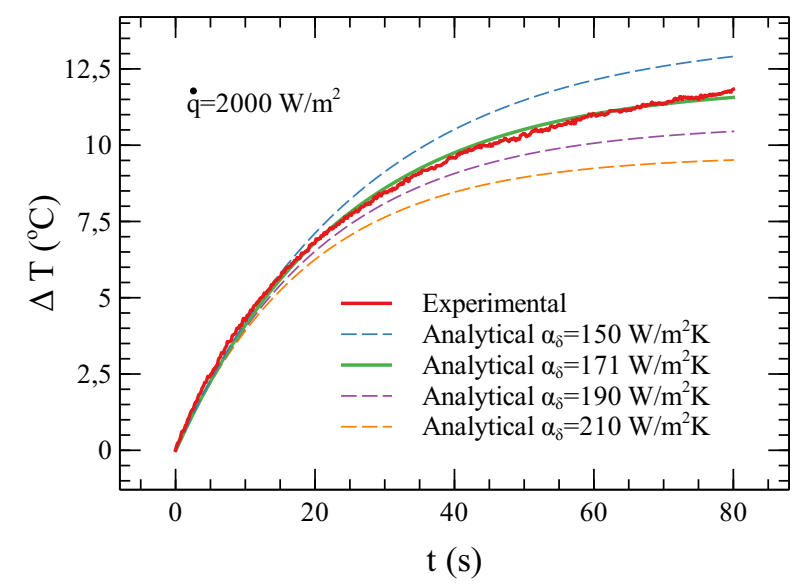

FiguRE 8. Comparison of experimental and analytical results. The optimization algorithm leads to the heat transfer coefficient $\alpha_{\delta}=171 \mathrm{~W} / \mathrm{m}^{2} \mathrm{~K}$.

of this system of equations, we used the Laplace's transformation to make the solution easier. An inverse transformation of the resulting equation is not easy, so we used a numerical method to perform the inverse Laplace transform.

The analytical results as well as the dependence of some thermophysical parameters of the wall were plotted. Other wall parameters do not have a major impact on the solution. We verified the correctness of our derivation by a numerical simulation in MATLAB.

Our idea of an experimental measurement was presented and also representative measured values of the wall temperature were compared with the analytical solution of this problem.

\section{LIST OF SYMBOLS}

a temperature diffusivity $\left[\mathrm{m}^{2} \mathrm{~s}^{-1}\right]$

$\mathrm{Bi}_{0} \quad$ Biot number in place $z=0\left(=\alpha_{0} \delta / \lambda\right)[-]$

$\mathrm{Bi}_{\delta}$ Biot number in place $z=\delta\left(=\alpha_{\delta} \delta / \lambda\right)[-]$

$C_{1}, C_{2}$ integration constants [-]

$c_{p} \quad$ specific heat capacity $\left[\mathrm{J} \mathrm{kg}^{-1} \mathrm{~K}^{-1}\right]$

$f(t)$ function in real time $t[-]$

$f^{\prime}(t)$ derivative of function $[-]$

$\bar{f}(s)$ image of the function in the Laplace space [-]

$H(t)$ Heaviside function $[-]$

$\dot{Q}$ heat flow rate $[\mathrm{W}]$

$\dot{q}$ heat flux $\left[\mathrm{W} \mathrm{m}^{-2}\right]$

$\hat{q}$ amplitude of heat flux $\left[\mathrm{W} \mathrm{m}^{-2}\right]$

$s$ variable in Laplace space [-]

$S$ surface $\left[\mathrm{m}^{2}\right]$

$t$ time [s]

$t^{\prime} \quad$ dimensionless time $\left(=a t / \delta^{2}\right)[-]$

$T$ temperature $\left[\mathrm{K},{ }^{\circ} \mathrm{C}\right]$

$T_{0}$ temperature at the time $t=0\left[\mathrm{~K},{ }^{\circ} \mathrm{C}\right]$

$T_{f}$ ambient temperature $\left[\mathrm{K},{ }^{\circ} \mathrm{C}\right]$

$\Delta T$ temperature difference $\left[\mathrm{K},{ }^{\circ} \mathrm{C}\right]$

$x, y, z$ coordinations [m]

$z^{\prime}$ dimensionless coordination $(=z / \delta) \quad[-]$

$\alpha$ heat transfer coefficient $\left[\mathrm{W} \mathrm{m}^{-2} \mathrm{~K}^{-1}\right.$ ]

$\alpha_{0}$ heat transfer coefficient in place $z=0\left[\mathrm{~W} \mathrm{~m}^{-2} \mathrm{~K}^{-1}\right]$

$\alpha_{\delta}$ heat transfer coefficient in place $z=\delta\left[\mathrm{W} \mathrm{m}^{-2} \mathrm{~K}^{-1}\right]$

$\delta$ thickness of the wall [m]

$\lambda$ thermal conductivity $\left[\mathrm{W} \mathrm{m}^{-1} \mathrm{~K}^{-1}\right]$

$\lambda$ roots of equation $[-]$

$\rho$ density $\left[\mathrm{kg} \mathrm{m}^{-3}\right]$ 


\section{ABbreviations}

EDM electrodiffusion method

RGB red-green-blue

TLC thermochromic liquid crystals

TOIRT temperature oscillation infra-red thermography

\section{ACKNOWLEDGEMENTS}

Authors acknowledge support from the Grant Agency of the Czech Technical University in Prague, grant SGS18/129/OHK2/2T/12 and support from the EU Operational Programme Research, Development and Education, and from the Center of Advanced Aerospace Technology (CZ.02.1.01/0.0/0.0/16_019/0000826), Faculty of Mechanical Engineering, Czech Technical University in Prague.

\section{REFERENCES}

[1] V. Katti, S. V. Prabhu. Experimental study and theoretical analysis of local heat transfer distribution between smooth flat surface and impinging air jet from a circular straight pipe nozzle. International Journal of Heat and Mass Transfer 51:4480-4495, 2008. DOI:10.1016/j.ijheatmasstransfer.2007.12.024.

[2] W. Kenneth, Z. Wang, P. T. Ireland, T. V. Jones. Detailed measurements of local heat transfer coefficient and adiabatic wall temperature beneath an array of impinging jets. In Proceedings of the International Gas Turbine and Aeroengine Congress and Exposition. The American Society of Mechanical Engineers, 1993.

[3] A. Beniaiche, A. Ghenaiet, C. Carcasci, B. Facchini. Heat transfer investigation in new cooling schemes of a stationary blade trailing edge. Apllied Thermal Engineering 87:816-825, 2015. DOI:10.1016/j.applthermaleng.2015.05.001.

[4] S. A. Hippensteele, P. E. Pinsatte. Transient liquid-crystal technique used to produce high-resolution convective heat-transfer-coefficient maps. In Proceedings of the National Heat Transfer Conference. Atlanta, Georgia, 1993.

[5] L. Bohm, S. Jankhah, J. Tihon, et al. Application of the electrodiffusion method to measure wall shear stress: Integrating theory and practice. Chemical Engineering and Technology 37(6):938-950, 2014. DOI:10.1002/ceat.201400026

[6] K. Petera, M. Dostál, M. Věříšová, T. Jirout. Heat transfer at the bottom of a cylindrical vessel impinged by a swirling flow from an impeller in a draft tube. Chem Biochem Eng Q 31(3):343-352, 2017. DOI:10.15255/CABEQ.2016.1057

[7] M. Cudak, J. Karcz. Studies of local heat transfer at vicinity of a wall region of an agitated vessel. In Proceedings of 34th International Conference of SSCHE. 2007.

[8] J. Karcz, F. Strek. Heat transfer in jacketed agitated vessels equipped with non-standard baffles. The Chemical Engineering Journal 58:135-143, 1995. DOI:10.1016/0923-0467(94)02945-8.

[9] M. Wandelt, W. Roetzel. Lockin thermography as a measurement technique in heat transfer. Quantitative Infrared Thermography 96 pp. 189-194, 1997. DOI:10.21611/qirt.1996.031.

[10] S. Freund. Local Heat Transfer Coefficients Measured with Temperature Oscillation IR Thermography. Ph.D. thesis, Universitat der Bundeswehr Hamburg, Hamburg, 2008.

[11] S. Freund, S. Kabelac. Investigation of local heat transfer coefficients in plate heat exchangers with temperature oscillation ir thermography and cfd. International Journal of Heat and Mass Transfer 53:3764-3781, 2010. DOI:10.1016/j.ijheatmasstransfer.2010.04.027.

[12] S. Freund, A. G. Pautsch, T. A. Shedd, S. Kabelac. Local heat transfer coefficients in spray cooling systems measured with temperature oscillation ir thermography. International Journal of Heat and Mass Transfer 50:1953-1962, 2007. DOI:10.1016/j.ijheatmasstransfer.2006.09.028.

[13] T. Myint-U, L. Debnath. Linear Partial Differential Equations for Scientists and Engineers. Borkhauser, 4th edn., 2007.

[14] J. Abate, W. Whitt. A unified framework for numerically inverting laplace transforms. INFORMS Journal on Computing 18(4):408-421, 2006. DOI:10.1287/ijoc.1050.0137.

[15] B. Weigand. Analytical Methods for Heat Transfer and Fluid Flow Problems. Springer, 2nd edn., 2015. 


\section{APPENDIX}

The MATLAB code for calculating the analytical value of the change of the temperature in the wall with the Talbot's inversion (variable definition, function definition and Talbot's inversion).

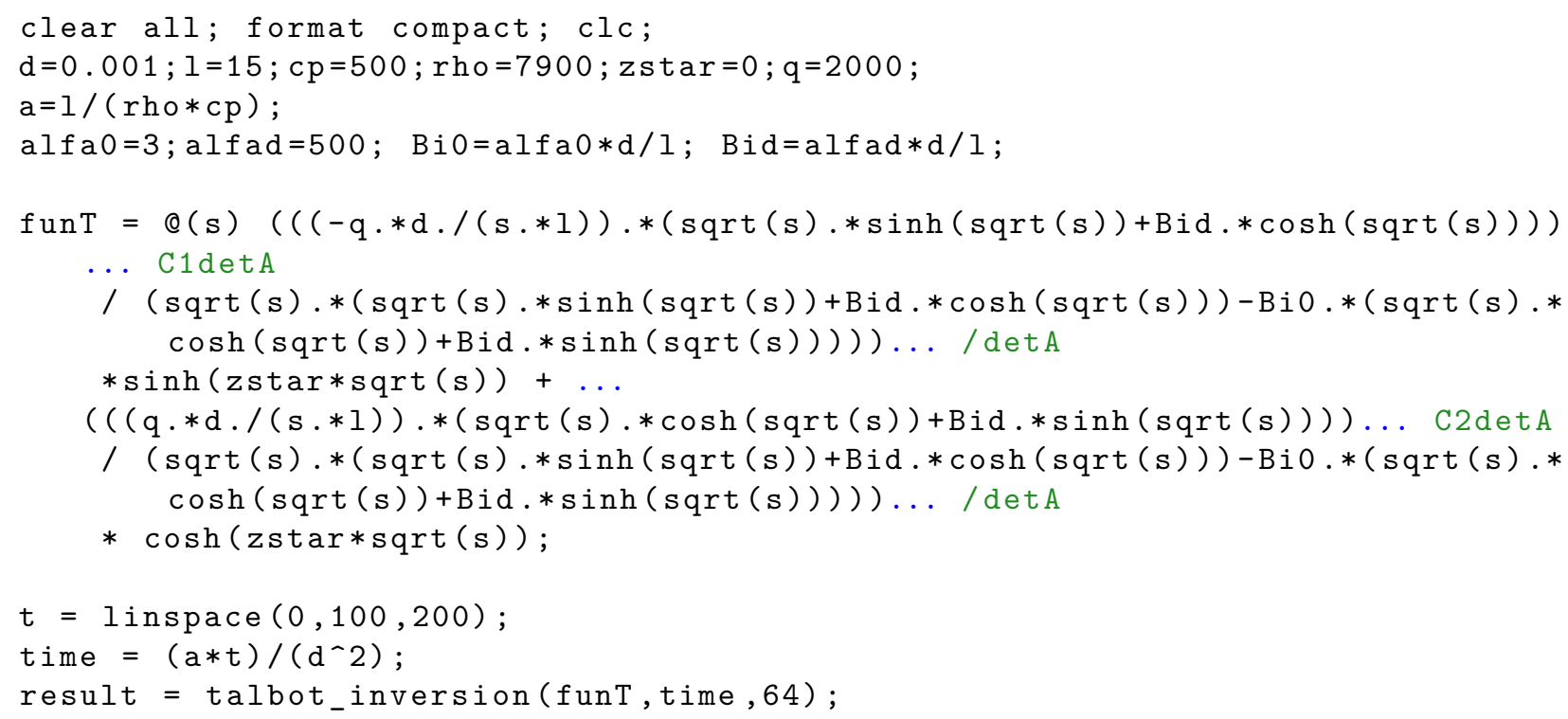

MATLAB code for numerical calculation of the change of temperature in the wall with the help of pdepe function (variable definition, spatial and time discretization, numerical calculation). The code is also supllemented with additional functions for writing a partial differential equation (pdefun), initial condition (icfun) and boundary conditions (bcfun).

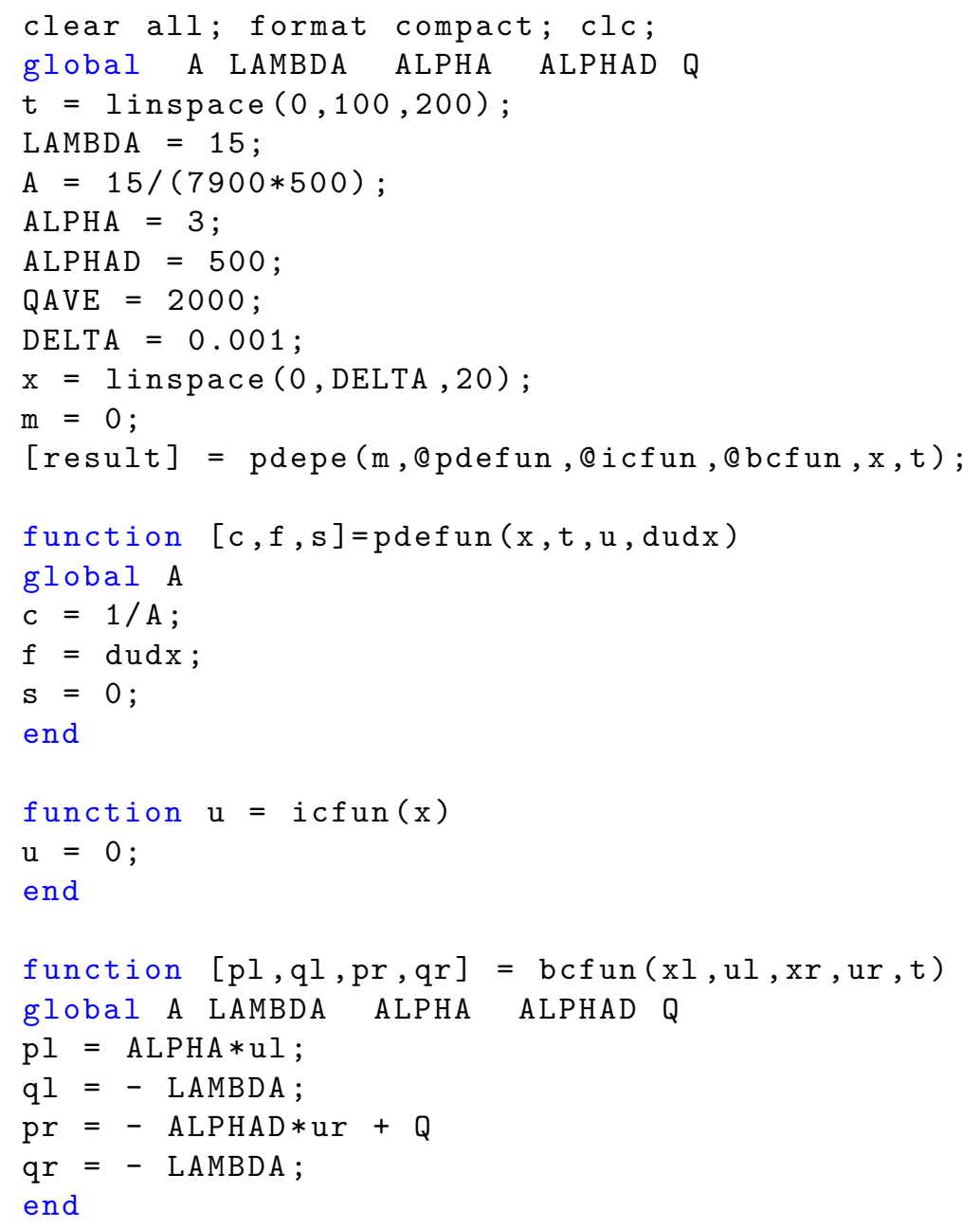

